\title{
The Importance of The Dental Hygienist in Implantology: A Narrative Review
}

Lucia Caporaso, Alessandra Bissoli, Francesca Iarussi, Riccardo Pulcini, Marco Dolci, and Silvia D'Agostino*

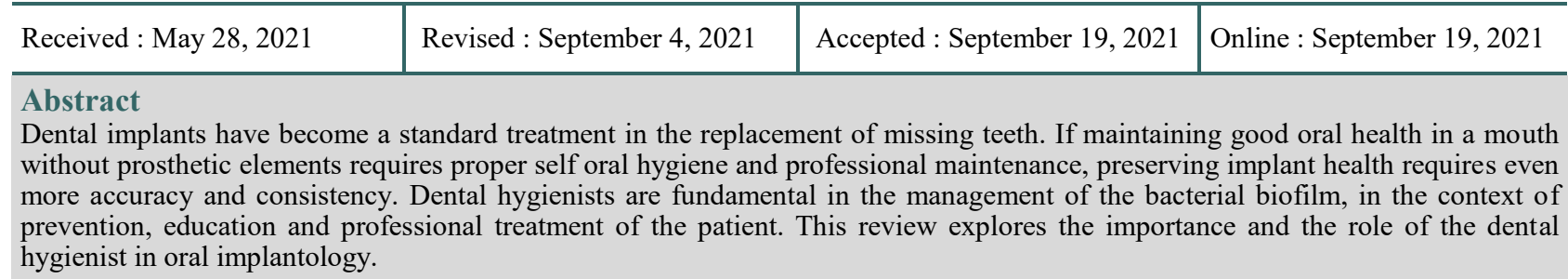

Keywords: biofilm, dental hygienists, dental implants, oral health, peri-implantitis

\section{INTRODUCTION}

Oral implantology employs osseointegrated implants used as support for fixed or removable prosthetic rehabilitation. It is universally recognized as a safe clinical methodology, able to ensure lasting results in oral therapy. Dental implants are similar but different from teeth. They lack some basic characteristic structures such as root cementum, periodontal ligament, and bundle bone. The implant doesn't have dento-alveolar and the dento-gingival fiber bundles that connect the soft tissues with the tooth (root cementum) and it is rigidly anchored (ankylosed) to the bone, whereas the tooth is mobile within its socket [1].

Before starting any dental implant therapy, clinicians must be thoroughly familiar with the patient's medical and oral condition and assess all the possible risks. Implant failure is not only related to poor osseointegration but also to the presence of some risk factors and conditions that influence the outcome and prognosis of implant therapy in the short, medium and long term [2]. Peri-implantitis is surely one of these conditions. As the 2017

Copyright Holder:

(C) Caporaso, L., Bissoli, A., Iarussi, F., Pulcini R., Dolci, M., and D'Agostino, S. (2022)

First Publication Right:

Journal of Multidisciplinary Applied Natural Science

Publisher's Note:

Pandawa Institute stays neutral with regard to jurisdictional claims in published maps and institutional affiliations.

This Article is Licensed Under:
World Workshop of Chicago 4th group stated, "peri-implantitis is a plaque-associated pathological condition occurring in tissues around dental implants, characterized by inflammation in the periimplant mucosa and subsequent progressive loss of supporting bone" [2]. Scientific evidence suggests that peri-implantitis may be secondary to periimplant mucositis resulting from poor home and professional maintenance [3]. So the main cause of this pathological condition, as for periodontitis, is the bacterial plaque also known as biofilm. Biofilm can be defined as a multi-microbial community immersed in a self-produced polymeric matrix, which associates with biotic or abiotic surfaces wetted by a liquid and it is the result of a multistage process. If in the early stages of bacterial colonization we have commensal bacteria, the biofilm composition changes as the plaque ages: in the deeper regions appear anaerobic bacteria. Even if biofilm associated with peri-implant disease has not been widely documented, the microbiome of healthy and diseased implants sites have been compared and peri-implantitis was associated with species of the red complex such as Porphyromonas gingivalis and Tannerella forsythia [4]. Other studies indicated that peri-implantitis was most frequently related to opportunistic pathogens such as Pseudomonas aeruginosa and Staphylococcus aureus [5][6], fungal organisms (e.g., Candida albicans) [5][7][8] and viruses (e.g., Human cytomegalovirus, Epstein-Barr virus) [9], suggesting a more complex and heterogeneous infection [10][11]. Achieving satisfactory control over bacterial biofilm in an environment that is typically colonized by a range of both harmful and 
non-harmful bacteria is not easy. It requires the commitment of various professionals and most importantly the total compliance of the patient, who must feel part of a project aimed at achieving lasting oral health. In particular dental hygienists work is about prevention and oral health maintenance. In fact, the dental hygienist is the key provider of preventive oral care (primary, secondary and tertiary prevention) in order to promote and improve the oral health of individuals, families and groups in society. He/She possesses the necessary professional qualifications, depending on the State, and is able to provide patient-centered, holistic and evidence based preventive oral care independently. Dental hygienists are routinely responsible for the patient's oral health education and maintenance of dental implants. Hygienists are asked to perform an educational and instructional task in order to achieve good compliance and to improve the patient's self hygiene behavior, a fundamental condition for maintaining optimal oral health. The aim of the study is to explore the importance and the role of the dental hygienist in the management of dental implants' biofilm.

\section{MATERIALS AND METHODS}

The literature search was performed on the PubMed database. Studies published in the last 5 years from 2015 to 2021, with free full text available, were considered for inclusion. The following keywords as MESH terms or free words were used: "dental hygienists" OR "dental hygiene" AND "dental implants" OR "dental hygienists" AND "dental implants". Both review articles and research articles considered relevant for discussion were selected and analyzed. Studies in other languages than English were excluded. Evidence was presented in the form of a narrative review. Study eligibility criteria were the role of the dental hygienist in the maintenance of dental implants through the connection between professional oral hygiene, motivation for home oral care, both performed by dental hygienists, and outcomes on implant maintenance.

\section{RESULTS AND DISCUSSIONS}

The selection process resulted in a total 101 potentially relevant articles. After review of titles and abstract, 97 articles were excluded because they didn't meet the eligibility criteria; The majority of the works didn't include the figure of the dental hygienist, so a total of 4 articles were reviewed. The work of Yong-Keum Choi et al. considered agestratified associations between dental health behaviors related to tooth brushing and oral hygiene product use in Korean adults with implants [12]. Authors found that self-performed oral hygiene practices combining tooth brushing, at least twice per day, and dental floss were found to be significantly related to a low prevalence of periodontitis in people with implants. Two other studies have focused on dental implant treatment and maintenance [13][14]; Both of them showed that mechanical debridement and oral hygiene instructions, performed by dental hygienists, were crucial in the treatment of peri-implant mucositis. In particular the second study suggests that clinicians need to check if the prosthetic construction allows access for optimal hygiene and it also underlines the importance of the follow up in patients with dental implants. Finally only one article by Schneider et al. discussed the frequency of visits to the dental hygienist and taylored oral hygiene measures in Switzerland [15]. No articles were found specifically addressing the role of the dental hygienist in the maintenance of dental implants.

Oral implantology is a young discipline, a field in which research still has a lot to do, especially in the maintenance of implant health and in the treatment of peri-implant pathologies. As studies have shown, maintaining good self-performed oral hygiene reduces the risk of peri-implant mucositis and peri-implantitis [12]-[14]. The explanation is that by frequently and carefully disaggregating the biofilm the most aggressive bacterial species are not allowed to proliferate, organize themselves and colonize the various niches of the oral cavity. A dental hygienist plays a key role in this: his goal should be to maintain peri-implant health even before therapeutic resolution. Success can be achieved through the awareness of the different types of implants, of the various oral hygiene tools, and of the patient's cleaning and maintenance skills. It is also necessary for the dental hygienist to become an integral part of the professional team composed by the implantology surgeon and the 
prosthetist to ensure that the prosthetic construction allows access for optimal hygiene. This allows the dental hygienist to discriminate and select the most suitable techniques and tools for patients and recommend tailored oral hygiene measures to increase the compliance. It is equally important that the patient understands, even before undergoing surgery, that the implant or the implants will require constant professional as well as self maintenance. Finally, there is an important factor that should not be underestimated in the study by C. Schneider et al. The visits to the dentist decreased slightly over the years, while the visits to the dental hygienist increased. The combination of both indicates a small increase in oral health care utilisation in Switzerland between 1992 and 2012. These observations point to a trend towards a more specialised and tailored approach in oral healthcare provision, which is underlined by the increasing ratio of dentist to non-dentist oral health care workers in other European countries [16][17].

\section{CONCLUSIONS}

Dental hygienists play a key role in maintaining implant health, managing patients' oral habits, and keeping patients' compliance high. However the lack of scientific studies investigating their valuable work on osseointegrated implants does not lead to definite conclusions. There is a need for further studies to evaluate the role of dental hygienists in oral implantology.

\section{AUTHOR INFORMATION}

\section{Corresponding Author}

Silvia D'Agostino - Department of Medical, Oral and Biotechnological Sciences, University G. d'Annunzio, Chieti - 65013 (Italy);

(D) orcid.org/0000-0002-4778-4768

Email: silviadagostino00@gmail.com

\section{Authors}

Lucia Caporaso - Department of Medical, Oral and Biotechnological Sciences, University G. d'Annunzio, Chieti - 65013 (Italy);

Alessandra Bissoli - Department of Medical, Oral and Biotechnological Sciences, University G. d'Annunzio, Chieti - 65013 (Italy);
Francesca Iarussi - Department of Medical, Oral and Biotechnological Sciences, University G. d'Annunzio, Chieti - 65013 (Italy);

Riccardo Pulcini - Department of innovative technologies in medicine \& dentistry, University G. d'Annunzio, Chieti - 65013 (Italy);

orcid.org/0000-0001-8334-0577

Marco Dolci - Department of Medical, Oral and Biotechnological Sciences, University G. d'Annunzio, Chieti - 65013 (Italy);

orcid.org/0000-0001-5495-5660

\section{REFERENCES}

[1] M. G. Araujo and J. Lindhe. (2018). "Periimplant health," Journal of Periodontology. 89: S249-S256. 10.1002/JPER.16-0424.

[2] T. Berglundh, G. Armitage, M. G. Araujo, G. Avila-Ortiz, J. Blanco, P. M. Camargo, S. Chen, D. Cochran, J. Derks, E. Figuero, C. H. F. Hämmerle, L. J. A. Heitz-Mayfield, G. Huynh-Ba, V. Iacono, K.-T. Koo, F. Lambert, L. McCauley, M. Quirynen, S. Renvert, G. E. Salvi, F. Schwarz, D. Tarnow, C. Tomasi, H.-L. Wang, and N. Zitzmann. (2018). Peri-implant diseases and conditions: Consensus report of workgroup 4 of the 2017 World Workshop on the Classification of Periodontal and PeriImplant Diseases and Conditions. Journal of Periodontology. 89 : S313-S318. 10.1002/ JPER.17-0739.

[3] F. O. Costa, S. Takenaka-Martinez, L. O. M. Cota, S. D. Ferreira, G. L. M. Silva, and J. E. Costa. (2012). Peri-implant disease in subjects with and without preventive maintenance: a 5-year follow-up. Journal of Clinical Periodontology. 39 (2): 173-181. 10.1111/j.1600-051X.2011.01819.x.

[4] G. R. Persson and S. Renvert. (2014). "Cluster of bacteria associated with periimplantitis". Clinical Implant Dentistry and Related Research. 783-793. 10.1111/ cid.12052.

[5] A. Leonhardt, S. Renvert, and G. Dahlén. (1999). Microbial findings at failing implants. Clinical Oral Implants Research. 10 (5): 339-345. 10.1034/j.16000501.1999.100501.x. 
[6] A. Mombelli and F. Décaillet. (2011). The characteristics of biofilms in peri-implant disease. Journal of Clinical Periodontology. 38 : 203-213. $\quad \underline{10.1111 / \mathrm{j} .1600-}$ 051X.2010.01666.X.

[7] F. Schwarz, K. Becker, S. Rahn, A. Hegewald, K. Pfeffer, and B. Henrich. (2015). Real-time PCR analysis of fungal organisms and bacterial species at periimplantitis sites. International Journal of Implant Dentistry. 1 (1): 9. 10.1186/s40729015-0010-6.

[8] M. Albertini, L. López-Cerero, M. G. O'Sullivan, C. F. Chereguini, S. Ballesta, V. Ríos, M. Herrero-Climent, and P. Bullón. (2015). Assessment of periodontal and opportunistic flora in patients with periimplantitis. Clinical Oral Implants Research. 26 (8): 937-941. 10.1111/ clr.12387.

[9] S. Jankovic, Z. Aleksic, B. Dimitrijevic, V. Lekovic, P. Camargo, and B. Kenney. (2011). Prevalence of human cytomegalovirus and Epstein-Barr virus in subgingival plaque at peri-implantitis, mucositis and healthy sites. A pilot study. International Journal of Oral and Maxillofacial Surgery. 40 (3): 271-276. 10.1016/j.ijom.2010.11.004.

[10] M. Rakic, M. Grusovin, and L. Canullo. (2016). The Microbiologic Profile Associated with Peri-Implantitis in Humans: A Systematic Review. The International Journal of Oral \& Maxillofacial Implants. 359-368. 10.11607/jomi.4150.

[11] M. Padial-Molina, J. López-Martínez, F. O'Valle, and P. Galindo-Moreno. (2016). Microbial Profiles and Detection Techniques in Peri-Implant Diseases: a Systematic Review. Journal of Oral and Maxillofacial Research. 7 (3). 10.5037/jomr.2016.7310.

[12] Y.-K. Choi and E.-J. Kim. (2020). Oral health status of Korean adults with implants according to their use of oral hygiene products: results from a nationwide population-based study (2013-2015). Journal of Periodontal \& Implant Science. 50 (4): 268. 10.5051/jpis.1904700235.

[13] J. Philip, M. L. Laine, and D. Wismeijer.
(2020). Adjunctive effect of mouthrinse on treatment of peri-implant mucositis using mechanical debridement: A randomized clinical trial. Journal of Clinical Periodontology. 47 (7): 883-891. 10.1111/ jepe.13295.

[14] S. Renvert, H. Hirooka, I. Polyzois, A. Kelekis-Cholakis, and H.-L. Wang. (2019). Diagnosis and non-surgical treatment of peri -implant diseases and maintenance care of patients with dental implants - Consensus report of working group 3. International Dental Journal. 69 : 12-17. 10.1111/ idj. 12490.

[15] C. Schneider, E. Zemp, and N. U. Zitzmann. (2019). Dental care behaviour in Switzerland. Swiss Dental Journal. 129 (6): 466-478.

[16] E. Widström, K. A. Eaton, and C. LuciakDonsberger. (2010). Changes in dentist and dental hygienist numbers in the European Union and Economic Area. International Dental Journal. 60 (4): 311-316. 10.1922/ IDJ-2530Widstrom06.

[17] P. Brocklehurst and R. Macey. (2015). Skillmix in preventive dental practice - will it help address need in the future? BMC Oral Health. 15 (1). 10.1186/1472-6831-15-S1$\underline{\mathrm{S} 10 .}$ 